\title{
Lyon la républicaine à la veille de la guerre de 1870 et des journées insurrectionnelles de la commune lyonnaise. 2 . Lyon, cité républicaine
}

Réédition de l'article paru dans 1848 - Revue des révolutions contemporaines, $\mathrm{n}^{\circ} 189$, décembre 1951, pp. 122-127

Mlle Bonnardel, J. Bouvier, M. Emerique et M. Moissonier

\section{(2) OpenEdition}

Édition électronique

URL : http://journals.openedition.org/rh19/258

DOI : $10.4000 /$ rh 19.258

ISSN : $1777-5329$

Éditeur

La Société de 1848

\section{Édition imprimée}

Date de publication : 1 juin 2001

ISSN : 1265-1354

Référence électronique

Mlle Bonnardel, J. Bouvier, M. Emerique et M. Moissonier, « Lyon la républicaine à la veille de la guerre de 1870 et des journées insurrectionnelles de la commune lyonnaise. 2. Lyon, cité républicaine ", Revue d'histoire du XIXe siècle [En ligne], 22 | 2001, mis en ligne le 04 septembre 2008, consulté le 30 avril 2019. URL : http://journals.openedition.org/rh19/258 ; DOI : 10.4000/rh19.258

Ce document a été généré automatiquement le 30 avril 2019.

Tous droits réservés 


\section{Lyon la républicaine à la veille de la guerre de 1870 et des journées insurrectionnelles de la commune lyonnaise. 2. Lyon, cité républicaine}

Réédition de l'article paru dans 1848 - Revue des révolutions contemporaines, $\mathrm{n}^{\circ} 189$, décembre 1951, pp. 122-127

Mlle Bonnardel, J. Bouvier, M. Emerique et M. Moissonier

Lyon, sous le Second Empire, est une ville républicaine, où légitimistes, gouvernementaux et tiers_parti sont incapables de s'entendre. Le mot d'ordre " le progrès par la liberté sans la révolution " proposée vainement en 1869 par l'Union Libérale et le journal Le Salut Public, masque mal les sentiments contradictoires des catholiques, inquiets pour le denier de saint Pierre mais plus inquiets encore pour le maintien de l'ordre.

Bloqués géographiquement dans les beaux quartiers que cerne du Nord et de l'Est le petit peuple, les éléments conservateurs sont politiquement paralysés.

Aussi Lyon est_elle représentée dès mars 1852 au Corps Législatif par un républicain : le Docteur Hénon. S'il refusa alors de prêter serment et ne siégea pas, il fut réélu en 1857, puis en 1863, et il est avec l'avocat Favre, l'un des Cinq.

La préfecture, qui en 1863 intervient avec prudence dans les élections, sera, en 1869, obligée de laisser faire, tant s'étaient révélés vains des efforts comme ceux rapportés en 1857 par un commissaire de police : " J'ai vu déjà les chefs des différents services du chemin de fer à la gare de Vaise, dont le personnel votera pour le gouvernement et ira au scrutin par brigades. Je me suis assuré de tous les crocheteurs du port, et du service des omnibus. J'ai vu aussi quelques industriels qui m'ont promis leur concours auprès de leurs ouvriers".

Rien n'y fit : la force du potentiel républicain à Lyon se mesure particulièrement aux élections de l'été 1867 au Conseil Général et au Conseil d'Arrondissement; dans le premier cas les républicains réunirent, les deux tiers des voix : 10804 sur 15817 votants ; dans le second, les trois quarts : 6193 sur 8757 votants.

Les bases populaires de républicanisme lyonnais 
Le républicanisme lyonnais est non seulement le résultat d'une tradition de luttes républicaines, mais en même temps l'émanation politique d'un. certain état de fait social. Le républicanisme lyonnais a une base populaire solide, dans les milieux ouvriers et dans les couches inférieures de la bourgeoisie, ce qui explique sa force mais aussi sans doute ses divisions, ses contradictions, ses insuffisances.

La classe, ouvrière lyonnaise n'a jamais accepté l'Empire, d'autant moins que, aux époques de crise économique, et tout spécialement après 1866, la fabrique lyonnaise connaît des hauts et des bas, et que le niveau de vie des canuts est très sensiblement atteint.

Aux élections législatives de 1863 le commissaire de police du quartier des Chartreux (Croix_Rousse), pour expliquer la double élection républicaine du Docteur Hénon et de l'avocat Jules Favre, note:

image " Les ouvriers ont été des adversaires de la pire espèce ; infestés de doctrines socialistes, ils tendent au renversement de tout ordre ".

En 1867, on lit dans " un rapport politique et confidentiel " du mois de décembre : " On constate le prix excessif des loyers, des patentes et des impôts, la cherté du pain et de la viande [...] les populations demandent que les salaires soient augmentés, une diminution sur les denrées de première nécessité. la suppression des droits d'octroi, le rétablissement du conseil municipal ".

À la même époque se multiplient à la Croix_Rousse des placards comme celui_ci, arrachés par la police:

" Depuis quinze ans qu'un intrus a usurpé le pouvoir, vous gémissez dans la plus grande misère. Néanmoins, vous fiant aux promesses mensongères du bâtard, vous êtes restés calmes jusqu'à ce jour. Mais aujourd'hui, son despotisme n'a plus de bornes, et l'infâme tyran n'a pas craint de verser le sang français pour la cause exécrée des Jésuites et des calotins et de faire massacrer sans pitié nos frères d'Italie. Il ruine le pays pour envoyer de l'or au pape tandis que vos enfants meurent de froid et de faim.

" Peuple esclave, à quoi sert l'argent des impôts ? À faire égorger tes fils dans des guerres lointaines et inutiles. Réveille_toi, peuple insensé, et souviens_toi qu'il ne faut qu'une vague pour engloutir la barque ".

La misère ouvrière alimente donc l'opposition politique au régime, comme elle exaspère le conflit des classes ; le " rapport politique et confidentiel " déjà cité le souligne sans ambages :

" L'union entre les négociants et les ouvriers n'existe pas. Il n'y a aucune sympathie entre ces deux classes. L'ouvrier ne cache pas son idée de vengeance contre le fabricant qui le fait travailler, et ce dernier, par réciprocité, ne favorise en rien le tisseur [...] Même les sociétés coopératives ouvrières peuvent devenir dangereuses pour la tranquillité du pays ".

Se devinent ici les motifs comme les limites du soutien gouvernemental à ces coopératives susceptibles d'enliser le mouvement ouvrier naissant.

La composition du public des réunions permet de vérifier que les tendances républicaines ont leur base populaire essentielle dans la classe ouvrière. Lorsque, le 12 avril 1869, le républicain Bancel vient faire à Vaise une conférence, le commissaire de police du quartier photographie ainsi la salle : " Le public se composait en grande partie d'ouvriers, qui étaient venus là comme à une fête, revêtus de leurs meilleurs habits ; de beaucoup d'employés de chemin de fer de Vaise, et d'environ cent femmes d'ouvriers qui étaient là avec leurs maris et leurs petits enfants ". 
Le républicanisme plonge ses racines dans d'autres milieux sociaux : dans la " boutique ", chez les petits et moyens industriels, voire même certains milieux d'affaires. Toutes ces catégories, à des degrés divers, souffrent des crises, sont les victimes de la concentration, critiquent, avec le système des impôts, la cherté des loyers, et cristallisent leur mécontentement contre les expéditions militaires et les dépenses gouvernementales. Le Préfet du Rhône lui_même, dans son rapport de juillet 1868 au ministre de l'Intérieur doit reconnaître que :

" [...] le petit commerce ne résiste que difficilement à la concurrence ruineuse des grandes maisons et à l'énormité des frais généraux, notamment du loyer ; l'échéance du terme de la Saint_Jean vient de confirmer cette situation, en lui donnant un caractère de sérieuse évidence : un grand nombre de déménagements ayant eu lieu par suite de cessation de commerce [...] Les petits commerçants ne font pas un chiffre d'affaires suffisant. Ils sont mal payés et obligés de faire de longs crédits ".

Scellant une solidarité de fait entre prolétaires et classes moyennes, la misère ouvrière est à la base du marasme de la boutique.

Certains milieux d'affaires eux_mêmes marquent leur hostilité au régime. Du moins le Préfet du Rhône (rapport au ministre de l'Intérieur du 19 septembre 1868) note_t_il, parmi les candidats possibles de ce qu'il appelle lui_même " la bourgeoisie libérale " : Arlès_Dufour, le gros négociant, ex_saint_simonien et encore romantique, et Henri Germain lui_même, le fondateur du Crédit Lyonnais.

Scission des républicains en modérés et radicaux

$\mathrm{Si}$, sous l'Empire, les ouvriers lyonnais votent pour les bourgeois républicains, le commissaire spécial Bergeret, dont les " mouches " s'infiltrent un peu partout, précise, lors de la campagne électorale de 1863 : " On disait hautement, dans un certain nombre d'ateliers, que les " Cinq " ne valaient pas mieux que les autres [...] que la cause des ouvriers ne peut être bien défendue que par un ouvrier ".

Les ouvriers lyonnais ne se font guère illusion. Du moins une partie d'entre eux, sur les vertus politiques et républicaines de leurs représentants. Ils votent pour Hénon et Favre faute de candidatures ouvrières, et afin de faire pièce à l'Empire.

Le fait est remarquable : le républicanisme lyonnais se scinde en deux sous l'Empire libéral, et la tendance " radicale ", " irréconciliable ", " révolutionnaire ", l'emporte finalement sur la tendance " libérale ", " modérée ", " bourgeoise ".

La scission dans le personnel politique républicain ne peut s'expliquer par les seules ambitions personnelles, rivalités de coteries ou oppositions de tempérament, motifs qui interviennent comme aliments de la rivalité politique plutôt que comme éléments déterminants de cette rivalité.

La division entre modérés à la Hénon et radicaux à la Bancel traduit, semble_t_il, les difficultés et les hésitations des couches moyennes lyonnaises, dont les républicains sont l'émanation. Économiquement, elles sont solidaires du prolétariat : la prospérité de la boutique n'est_elle pas liée au pouvoir d'achat ouvrier? Politiquement, cette solidarité se marque par le soutien des ouvriers aux républicains bourgeois lors des élections. Mais, dans la mesure précisément où ce soutien est mis en cause par l'évolution politique du prolétariat, par une prise de conscience de classe, favorable à, la naissance d'un mouvement politique ouvrier qui ne serait plus à la remorque du républicanisme bourgeois, l'hésitation se fait jour chez les républicains bourgeois devant l'aventure sociale que, désormais, devient tout changement politique. Le langage démocratique, la phrase sentimentale, le verbalisme anti_impérial, avec lesquels ont jonglé jusqu'alors les avocats diserts des Loges, ne suffisant plus à entraîner les masses en les disciplinant. La 
peur du peuple, toujours latente, les souvenirs des " désordres ", des batailles de rues et de l'état de siège qui les suit, la crainte de la violence nécessaire au renversement de l'ordre " légal ", tout un complexe de réflexes et de réflexions paralyse une partie des républicains.

Aussi, tout en ayant des programmes politiques fort voisins, les deux tendances de la bourgeoisie républicaine lyonnaise s'éloigneront_elles chacune davantage l'une de l'autre, à mesure que s'approfondiront les contradictions du régime impérial, à mesure que grandira le divorce entre le gouvernement et la nation ; les modérés à la Hénon finissant par faire corps avec le régime, les radicaux à la Bancel acceptant le risque d'un bouleversement. Car un 1830 ne semble plus possible, 1848 l'a bien prouvé. Une révolution politique représente un risque qui se mesure à la maturité de la classe ouvrière. Si Bancel et ses amis pensent rester les maitres du mouvement, la crainte d'une conflagration sociale, qui irait bien au_delà d'un simple changement d'étiquette politique, retient au contraire Hénon et Jules Favre.

\section{Les deux équipes républicaines}

Premier trait remarquable de la vie politique lyonnaise à la fin de l'Empire, les politiciens républicains sont uniquement recrutés dans la petite et moyenne bourgeoisie, alors que la masse des électeurs républicains est ouvrière ; par ailleurs, l'organisation républicaine dans les quartiers et les arrondissements est entre les mains de petits états_majors " de boutiquiers et de chefs d'ateliers " (expression d'un " radical " en 1864), petits états_majors de notabilités de quartier que personne ne surveille ni ne contrôle d'en bas, et qui n'ont de rapport avec la population, et de vie politique véritable, qu'au moment des élections diverses. Enfin, la franc_maçonnerie fournit les cadres du républicanisme lyonnais au point que les comités républicains d'arrondissement méritent d'être appelés les " Petites Loges " ; les rites, le cérémonial, l'atmosphère secrète, les signes de reconnaissance francs_maçons se retrouvaient dans l'organisation républicaine. Tous les " frères " lyonnais se recrutaient d'ailleurs dans les milieux bourgeois : médecins, avocats, négociants, propriétaires, rentiers...

À côté de ces traits communs, les deux équipes républicaines se différencient assez nettement. La tendance modérée, bourgeoise, libérale, a pour chef de file Hénon ; le Docteur Hénon a 67 ans en 1869 ; étudiants en médecine de vieille famille lyonnaise, il a participé à Paris aux Trois glorieuses; il entre dans la vie publique en 1843 comme conseiller municipal des Brotteaux ; c'est un homme réservé, un érudit. Ce bourgeois très. respectueux des formes, sans grand contact avec ses mandataires, se considère rapidement comme député à vie, volontiers pontifiant.

À ses côtes, l'avocat Jules Favre, plus parisien que lyonnais, se montre légaliste, très " constitutionnel ", d'esprit religieux et volontiers philosophique.

Autour des deux hommes, les notables du Comité Central Démocratique : Bonnardel, Varambon, Saunier, Laforest, Frédéric Morin, Ferrouillat, Crestin... Agents de change, avocats, médecins, et tous francs_maçons. Mais, comme le dit Mlle R. Bonnardel " désirant plus l'élargissement des libertés parlementaires que l'avènement d'une République à l'avenir plein de menaces socialistes ".

Une lettre d'Ernest Picard et de Carnot, envoyée aux émigrés républicains de Londres, impatients de l'insurrection libératrice, porte témoignage de la tiédeur du républicanisme à la Hénon :

" Il n'est pas généreux d'exciter des tempêtes dont nous, vos amis politiques, serions les premières victimes [...] Si malgré nos observations vous persistez dans votre projet si compromettant pour nous, nous nous verrons forcés, pour dégager notre responsabilité, 
de mettre entre les mains de qui de droit votre correspondance ". (Archives Municipales lyonnaises, T 246 ; lettre du 6 juillet 1864).

$\mathrm{Au}$ Comité Central Démocratique furent attirés, il faut le signaler, quelques ouvriers sans doute flattés de telles fréquentations, comme Baudy (ami du docteur Crestin) emprisonné quelque temps après l'attentat d'Orsini ; Baudy, dit Crestin, " avait beaucoup réfléchi en prison ". Par lui et ses compagnons, les modérés essayeront non sans quelque succès d'intervenir dans les affaires des Internationalistes Lyonnais.

La tendance radicale est organisée autour du Comité Central Radical : Président, le peintre Chaverot ; Secrétaire, le relieur Favier ; avec eux Sébastien Commissaire, la plus forte personnalité du Comité, ex_législateur de la Seconde République, et ses amis : Anjou, l'avocat Louis Andrieux, Duguerry, Rivoire, Grosbois, Faure, Langlade, le chirurgien Barrier. " Prêts à faire cause commune avec le socialisme sans en connaître au juste les revendications " (G. Weill), il y a là, côte à côte, de vieux " quarante-huitards ", des ex_condamnés politiques rentrés après l'amnistie, et une équipe de jeunes bourgeois, dont l'ambition n'était pas sans doute le dernier mobile.

Au moins une partie des radicaux et surtout Sébastien Commissaire et ses amis sont les héritiers du courant montagnard de 1849, avec tout ce que ce courant représente alors de confusion, d'impuissance, voire d'hypocrisie. Les souvenirs de 1849 à Lyon expliquent certainement que les ouvriers votent radical en masse ; le radical, qui se réfère habilement à la victoire montagnarde aux élections de la législative à Lyon, sait parler à ses électeurs prolétariens ; il en est entendu, il se solidarise avec eux. Serait révélateur, à ce propos, l'examen du passé politique de Sébastien Commissaire. Mais en 1868_1869 celui_ci s'efface comme futur candidat des radicaux qui font appel, sur ses conseils, à des non_lyonnais.

Ce fut habileté et non pas maladresse de leur part que de prendre comme porte_parole aux élections de 1869, deux " étrangers " de marque : le médecin Raspail, unanimement connu et adoré des masses populaires, le méridional docteur des pauvres, âgé de 75 ans en 1869, toujours " apôtre du camphre ", débordant d'éloquence, de bonté, de générosité vraie, incarnant magnifiquement aux yeux des ouvriers lyonnais, cette " République sociale " idéale et vague qu'ils appelaient de leurs vœux depuis le temps des fusillades. À ses côtés l'ex_législateur Bancel, de Valence, proscrit du 2 décembre qui avait refusé de rentrer en France à la faveur de l'amnistie, et qui vivait de leçons de littérature données l'Université de Bruxelles. Dès le printemps de 1867, Sébastien Commissaire était allé le chercher pour le fléchir.

Bancel vint en 1868 faire des conférences à Lyon ; revint en 1869 pour une nouvelle tournée, et prépara ainsi sa campagne électorale dans les quartiers populaires, sous l'œil vigilant des commissaires de police, présents dans la salle ; celui de Vaise qui avait si bien noté la composition ouvrière du publie, analysait ainsi le contenu de la prédication de Bancel, le 12 avril 1869 :

" Il a déclaré que le point primordial était l'instruction, qu'un peuple n'était pas digne d'avoir la liberté s'il ne possédait pas d'abord. l'instruction. Il s'est élevé contre l'autocratie des castes religieuses qui, dans tous les âges, ont asservi l'instruction en la faisant toujours tourner au profit de leur influence et de leur ambition [...] Il a terminé en formulant l'espoir de voir un jour la France, un jour plus près qu'on ne le pense, dotée de l'enseignement obligatoire [...] Il a fait de nombreuses allusions à l'indépendance des peules d'une manière fugitive, habile [...] Cependant, j'ai remarqué sa phrase finale : " Ainsi, dans peu de temps, il faut l'espérer, plus de couvents, peu de casernes, et beaucoup d'écoles! " 


\section{Programmes républicains}

Entre modérés et radicaux, la fêlure s'agrandit progressivement de 1863 à 1869, à mesure que les problèmes politiques se posent avec une netteté de plus en plus grande. Vient un moment où il ne suffit plus de prendre parti en paroles, mais de préparer l'action. Ce moment, à Lyon, se situe à l'automne 1868, lorsque Le Progrès radical (après d'ailleurs nombre d'hésitations et de manœuvres diverses) et La Discussion se trouvent en désaccord sur un point précis de tactique électorale. Dans le Var, en effet, des élections partielles opposent Dufaure à un officiel. Or, qui est Dufaure? Un monarchiste, un clérical ; qui plus est, ancien ministre de l'Intérieur de juin 1849, il avait proclamé l'état de siège à Paris et Lyon lors de la poussée " montagnarde ", emprisonné les républicain livrés aux tribunaux militaires, suspendu les libertés de réunion et de presse. Bref, disait Le Progrès, " le proscripteur de juin 1849 ".

Tandis que les radicaux lyonnais proposaient l'abstention aux électeurs varois, et s'opposaient avec violence au politicien réactionnaire et clérical Dufaure, les Cinq à Paris, Hénon et ses amis à Lyon, disaient, au contraire : " Pour battre le candidat officiel, votez à droite, votez Dufaure ".

Certains compagnons de Hénon, comme le docteur Crestin, n'acceptèrent pas les ordres des Cinq et marchèrent, avec les radicaux, contre Dufaure... Ce dernier fut battu : il recueillit 12800 voix, le candidat officiel 17 400. Mais il y eut 18000 abstentions, que les radicaux lyonnais s'annexèrent, non sans quelque raison d'ailleurs.

Dans ce combat préliminaire, Hénon et Favre perdirent la bataille des élections de 1869. Certes, la classe ouvrière avait depuis longtemps politiquement jugé Hénon ; en mars 1866, le commissaire spécial Bergeret écrivait que Hénon était " à peu près dépopularisé à Lyon " ; le 25 avril 1867, l'indicateur préfectoral notait " le mépris affiché pour Hénon et ses vieilles défroques libérales ". Mais l'affaire du Var fut fatale aux républicains modérés lyonnais ; le 20 avril 1869, le Préfet écrivait au ministre de l'Intérieur :

" Hénon perd beaucoup de terrain [...] quelques ouvriers et une partie de la petite bourgeoisie lui restent encore fidèles, mais la masse ouvrière s'éloigne de lui ; sa candidature parait actuellement très compromise ".

En vérité, les programmes des modérés et des radicaux, à les comparer, dénotent un grand air de parenté. La simple énumération des points communs aux deux tendances révèle leur fond conforme aux intérêts de la bourgeoisie :

$1^{\circ}$ Liberté de l'industrie et abolition des monopoles (revendication_type de la petite bourgeoisie, illustrée en 1869 par l'ouvrage de G. Duchéne, L'Empire Industriel).

$2^{\circ}$ Liberté politique, entendue comme fondement de toutes les autres libertés $3^{\circ}$ Décentralisation administrative.

$4^{\circ}$ Séparation de l'Église et de l'État.

$6^{\circ}$ Instruction gratuite obligatoire.

$6^{\circ}$ Nation armée.

$7^{\circ}$ Libertés municipales.

On, retrouve là, sans surprise, une bonne partie du programme de Belleville.

Les deux questions sur lesquelles s'affrontent semblent s'affronter les deux tendances, ne sont pas en réalité, tellement déterminantes : les radicaux font profession d'anticléricalisme agressif, les modérés sont beaucoup plus modérés à ce sujet dans leur langage. Les radicaux sont, se disent, " sociaux ", les modérés ignorent bien davantage le prolétariat. 
Encore faut_il y regarder de plus près. Pour La Discussion, il n'y a pas de question sociale : " Nous ne sommes pas indifférents aux questions sociales ; mais ce qui nous déplaît dans ce terme, c'est qu'il est vague. Nous appelons questions sociales toutes les questions qui intéressent la collectivité [...] Or on l'applique le plus souvent aux réformes qui intéressent les classes ouvrières. Nous n'apercevons pas, quant à nous, pourquoi on mettrait à part cette catégorie de réformes ".

Les radicaux parlent d'or, au contraire, quand Le Progrès (28 juillet 1867) écrit : " Dans une ville comme la nôtre, où l'élément travailleur domine, l'affranchissement et l'émancipation de l'ouvrier peuvent_ils véritablement se réaliser sans de sérieuses réformes sociales marchant à l'unisson des réformes politiques ?"

En réalité, radicaux et modérés de concert avaient dès 1867 vivement critiqué l'essai tenté par les internationalistes lyonnais de présenter des candidats ouvriers aux élections du Conseil Général, mais la circulaire de Bancel dit, en 1869 :

" La question politique et les problèmes sociaux ne peuvent se résoudre que par la libre initiative des citoyens, en dehors de toute direction et de toute faveur de l'État ". De fait, les radicaux n'ont pas une notion plus précise que les modérés des questions ouvrières et se contentent d'un vague sentimentalisme sans conséquence. Ces politiciens ont seulement besoin des voix ouvrières ; la clientèle fait pression sur le candidat, exige des engagements, et le candidat accommode son programme en conséquence; en ce domaine, les radicaux l'emportaient aisément sur les modérés et se taillaient de gros succès d'auditoire par des tirades sur l'inégalité des richesses.

" Ce qui frappe le plus constamment dans les différents programmes politiques radicaux, écrit très justement Mlle Bonnardel, c'est qu'aucune place n'est faite à la question sociale, laquelle se pose pourtant en 1869 avec une extrême gravité et une grande urgence. Si les ouvriers lyonnais affirment leur sympathie pour les radicaux avec un si bel ensemble, c'est parce qu'ils sont persuadés et ils veulent l'être que la république radicale, en leur apportant la liberté politique, ne saura manquer de se préoccuper de leur malheureux sort et se fera une obligation de satisfaire aux revendications légitimes de ceux qui auront été les artisans de son avènement. C'était se faire de bien cruelles illusions : tous les candidats radicaux, issus de la bourgeoisie libérale, n'avaient aucun projet précis de politique sociale. pour cette raison qu'ils n'avaient guère pensé qu'il put y avoir une question sociale à la fin du Second Empire, et parce que cette " vile multitude " dont ils se servaient pour se faire élire, ne leur inspirait au fond, que crainte et mépris, peut_être sans qu'ils se l'avouassent eux_mêmes ". On entrevoit déjà ce que sera l'attitude radicale et celle, à plus forte raison, de l'ensemble de la bourgeoisie républicaine quand viendront avec 1870 , l'activité politique concrète, des masses, et le spectre du " quatrième état ".

En attendant, et à la veille des élections de 1869, les radicaux s'annonçaient comme des adversaires irréconciliables de l'Empire, à la différence d'Hénon et de son état_major. Mais la tendance radicale ne devait pas tant à elle_même son succès qu'au manque de maturité des couches populaires lyonnaises.

Les ouvriers anglais, dans ces années, n'étaient pas les seuls à voter " haut de forme ". 
INDEX

Mots-clés : Elections, Histoire politique, Histoire sociale, Opinion publique, République, Second empire 